\title{
Las propiedades relacionales importan: transhumanismo, ciencia ficción y el problema de la identidad personal
}

\section{doi: 10.52749/fh.v2i3.5}

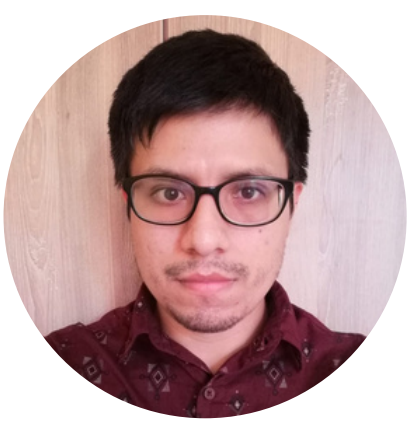

\section{JULIO SILVA CÉSPEDES iD https://orcid.org/0000-0002-0931-7528}

Estudiante de Filosofía en la Universidad Nacional Mayor de San Marcos. Miembro del instituto de extrapolítica y transhumanismo (IET) y del grupo de investigación Sentido y Referencia (UNMSM). Principales áreas de investigación: historia de la filosofía, filosofía de la mente, lógica y ética. Ha expuesto sus trabajos en eventos como el XVII Congreso Nacional de Filosofía (2019), el Primer Simposio Internacional de Lógica (2019) y el VIII Coloquio de Filosofía Latinoamericana (2020).

\section{julio.silva9@unmsm.edu.pe $\triangleright$ Filosofarte}

Resumen. En este ensayo, evaluaremos el problema de la identidad personal en el contexto del transhumanismo. En primer lugar, en contra de la tesis de que el transhumanismo representa una amenaza a la persistencia de la identidad personal argumentaremos lo siguiente: los individuos humanos que son personas no tienen características esenciales. En segundo lugar, con la ayuda del primer episodio de la segunda temporada de la serie de ciencia ficción Black Mirror, defenderemos la tesis de que hay propiedades que realmente importan en el problema de la identidad personal: las propiedades relacionales (esta tesis tiene como consecuencia que nuestros seres queridos son irremplazables, incluso por duplicados exactos). Finalmente, concluiremos que el uso de las tecnologías de mejoramiento humano no representa una amenaza a la identidad personal de un individuo en tanto que no altera sus propiedades relacionales importantes.

Palabras clave: identidad personal, filosofía, mejoramiento humano, propiedad relacional, transhumanismo.

\section{Transhumanismo y el problema de la identidad personal}

El transhumanismo es un movimiento filosófico y político que promueve el uso de tecnologías que permiten el mejoramiento de las capacidades físicas e intelectuales. Así, los defensores del transhumanismo esperan que en el futuro estas tecnologías nos permitan superar las limitaciones impuestas por nuestras actuales capacidades. Podríamos, por poner solo algunos ejemplos, controlar el proceso de envejecimiento, podríamos mejorar y expandir nuestros sentidos, nuestra memoria, etc.

Hay dos aspectos del transhumanismo: un aspecto descriptivo y un aspecto normativo. El aspecto descriptivo señala que actualmente ya hay tecnologías de mejoramiento humano que se usan (píldoras para mejorar la memoria, ingeniería genética, medicina antienvejecimiento, etc.) y se seguirán desarrollando tecnologías que nos permitirán superar las actuales limitaciones humanas (nanotecnología molecular, superinteli- gencia, interfaz humano-computadora, etc.). El aspecto normativo señala que el uso de estas tecnologías es moralmente correcto y que deben continuarse e incentivarse. En este ensayo no discutiremos el aspecto normativo del transhumanismo. Para un panorama general del debate sobre el uso de las tecnologías del mejoramiento humano, el lector puede remitirse al libro Human enhancement [EI mejoramiento humano], editado por Bostrom y Savulescu (2009).

Ahora, sean o no realistas las expectativas del transhumanismo, lo cierto es que estas posibilidades dan lugar a preguntas filosóficas en torno al problema de la identidad personal. ¿Cómo así? Diversas razones se pueden ofrecer en contra del transhumanismo (razones éticas, religiosas, políticas, etc.). Pues bien, una de las objeciones contra el proyecto transhumanista podría ser el siguiente argumento que discute DeGrazia (2005, p. 269):

Premisa 1: una tecnología $\mathrm{X}$ de mejoramiento altera 
la identidad de una persona.

Premisa 2: alterar la identidad de una persona es altamente problemático.

Conclusión: la tecnología $X$ de mejoramiento es altamente problemática.

Este argumento en contra del transhumanismo se basa en la premisa de que las tecnologías de mejoramiento alteran la identidad de una persona. Es decir, si una persona se sometiera a procedimientos que mejoran radicalmente ciertas características propias, ¿cómo podríamos estar seguros de que la persona resultante seguiría siendo la misma que antes de tal procedimiento? Después de todo, el objetivo de las tecnologías de mejoramiento humano es cambiar las capacidades físicas y mentales de los individuos, y esto puede cambiar rasgos propios de tales personas. Así, algunos filósofos sostienen que el uso de tecnologías del mejoramiento humano involucra, en gran medida, un suicidio que trae a la existencia a una nueva persona (Elliott, 1999, pp. 28-29):

"Lo que es preocupante sobre las así llamadas "tecnologías de mejoramiento" podría no ser el prospecto del mejoramiento, sino el hecho más básico de alterarse a uno mismo, de cambiar capacidades y características fundamentales de la identidad de uno. (...) [Profundas] preguntas parecen estar en disputa cuando hablamos sobre el cambiar la identidad de una persona, el núcleo mismo de lo que una persona es. Hacer que alguien sea más inteligente, darle una nueva personalidad o incluso darle un nuevo rostro (...). Estos [cambios] significan, en algún sentido, transformar a ese individuo en una nueva persona."

Es importante notar que este tipo de argumento descansa en la asunción de que los individuos tenemos características esenciales, es decir, características que nos son propias y sin las cuales no seríamos los individuos que somos. Así, el argumento discutido por DeGrazia se puede reformular de la siguiente manera:

Premisa 1: el uso de tecnologías radicales de mejoramiento altera las propiedades esenciales de una persona.

Premisa 2: alterar las propiedades esenciales de una persona destruye a esa persona $(y$, a la luz de nues- tra discusión, origina a una nueva persona).

Conclusión: el uso de tecnologías de mejoramiento radical es un suicidio que, paradójicamente, trae a la existencia a una nueva persona.

Una primera objeción es que este argumento confunde dos sentidos de identidad, a saber, la identidad numérica y la identidad diacrónica. Por un lado, la identidad numérica es la relación de identidad que todo objeto tiene solo consigo mismo; por ejemplo, en este momento, la persona que escribe este ensayo y Julio Silva son numéricamente idénticos, puesto que son una y la misma persona. Por otro lado, la identidad diacrónica es el sentido de identidad como persistencia que un objeto tiene a lo largo del tiempo (Silva, 2021). Este último es el sentido de identidad que tenemos en mente cuando decimos que, por ejemplo, ahora mismo soy la misma persona que era hace cinco años, incluso aunque podría haber cambiado en varios aspectos. El argumento presentado no distingue estos dos sentidos de identidad, puesto que la premisa (1) es plausible solo si se refiere a las propiedades esenciales de la identidad diacrónica, mientras que la premisa (2) es plausible solo si se refiere a las propiedades esenciales de la identidad numérica. ¿Cómo así? Lo que sucede es que el cambiar nuestras propiedades esenciales no nos destruye si consideramos este cambio desde la identidad diacrónica (después de todo, esos cambios suceden a lo largo de nuestra vida, pues no tenemos las mismas propiedades que teníamos cuando éramos bebés, por ejemplo). Esta es la primera objeción que presenta DeGrazia (2005, pp. 264-266).

Ahora bien, interpretando el argumento de manera caritativa, podemos tratar de asumir que los individuos tienen propiedades esenciales o lo que Elliot (1999) denominó capacidades y características fundamentales de la identidad de uno. En ese sentido, las dos premisas del argumento presentado se interpretarían como que se están refiriendo a la identidad sincrónica y que esta se alteraría si se modifican las propiedades esenciales. Entonces, es legítimo plantear la pregunta de cuáles podrían ser esas posibles características y capacidades fundamentales de la identidad de un individuo humano.

\section{¿Tenemos propiedades esenciales?}

Elliott arguye que los mejoramientos radicales nos harían perder características que son esenciales 
para la identidad de uno mismo. ¿Cuáles podrían ser esas características esenciales? Una primera propuesta podría ser que la característica esencial de una persona es su personalidad psicológica. Sin embargo, esta respuesta es problemática: un individuo humano cambia de personalidad a lo largo de su vida. No hay una personalidad fija. Además, hay tratamientos psiquiátricos que, precisamente, apuntan a cambiar la personalidad de un individuo, y difícilmente alguien objetaría que estos tratamientos, al cambiar la personalidad, son problemáticos. Si los tratamientos psiquiátricos no son problemáticos, ¿por qué lo sería el uso de tecnologías de mejoramiento de cualidades psicológicas? Después de todo, el transhumanismo propone añadir o mejorar ciertas cualidades psicológicas y, en ese sentido, nada sería perdido (o al menos nada realmente valorable).

En este punto, se podría objetar que, a pesar de que la personalidad de un individuo humano cambia a lo largo de su vida, esa persona no deja de ser humano, es decir, no deja de pertenecer a la especie Homo sapiens. Así, el transhumanismo es problemático porque sugiere que los humanos podríamos cambiar tanto que probablemente nos convirtamos en una especie diferente (posthumanos), lo cual es problemático. Entonces, la segunda propuesta sería que la característica esencial de una persona es pertenecer a la especie Homo sapiens y, en ese sentido, se debe preservar la especie humana (no se deben usar las tecnologías de mejoramiento). Esta propuesta la podemos encontrar en el escrito de los bioconservadores George Annas, Lori Andrews y Rosario Isasi (2002). En ese entonces, ellos promovían la creación de una 'Convención sobre la preservación de la especie humana'.

Ahora bien, esta última propuesta también es problemática por las siguientes razones: a) el concepto humano (animal) es diferente del concepto persona, puesto que ser persona es una propiedad del animal humano (Villena , 2010, pp. 6667): las características de una persona son ser autoconsciente (consciente de sí mismo), consciente (de los objetos externos a la persona), racional, etc., y todas estas cualidades las puede tener o no el animal humano; b) no hay razón para darle importancia a la pertenencia a una especie: si conociéramos alienígenas que comparten nuestra inteligencia, identificaríamos características similares a las nuestras, por ejemplo, la auto- consciencia, la racionalidad, etc.; c) es muy controversial el problema filosófico de qué es una especie:

\begin{abstract}
"A menudo los biólogos discuten lo que ellos llaman 'el problema de la especie'. Este se refiere al problema de dar una definición precisa de lo que es una especie. Muy sorprendentemente, no hay un consenso sobre este asunto. (...) La razón [de que una especie no sea algo fijo] es simple: en cada especie, encontramos variaciones considerables entre sus organismos constituyentes. Las mutaciones continuamente arrojan nuevas variantes genéticas, y la reproducción sexual continuamente 'baraja' los genes, resultando en diferencias genéticas extensivas entre los organismos dentro de una misma especie. Además, la constitución genética de cualquier especie cambia a lo largo del tiempo, a medida que evoluciona" (Okasha, 2019, pp. 81-83).
\end{abstract}

En ese sentido, usemos o no las tecnologías de mejoramiento humano, inevitablemente la 'especie' Homo sapiens cambiará a lo largo del tiempo, a menos que nos extingamos en un futuro no muy lejano. En consecuencia, no es posible que los individuos humanos tengan propiedades esenciales que sean fijas. Los individuos y las especies siempre cambian a lo largo del tiempo. Así, cualquier otra sugerencia que se proponga como una característica esencial de los individuos humanos tendrá dificultades. En un tono muy similar, el célebre biólogo y filósofo de la ciencia Ernst Mayr (2004, pp. 26-27) ha argumentado que las ideas esencialistas no son aplicables en la biología.

En síntesis, el argumento que acabamos de desarrollar es el siguiente:

Premisa 1: si los individuos que son humanos (y su especie) cambian a lo largo del tiempo, entonces no tienen propiedades esenciales.

Premisa 2: los individuos que son humanos (y su especie) cambian a lo largo del tiempo.

Conclusión: los individuos que son humanos no tienen propiedades esenciales.

Esta conclusión también la comparte DeGrazia (2005, pp. 272-280). Ahora bien, a pesar de esta conclusión aquí sostenemos que hay cualidades que 
realmente importan en el problema de la identidad personal: las propiedades relacionales. Desarrollemos esta idea.

\section{¿Reemplazarías a tu ser querido fallecido?}

Para defender la tesis de que las propiedades relacionales importan al abordar el problema de la identidad personal, usaremos como punto de partida una historia que se muestra en el episodio Ahora mismo vuelvo - Be Right Back, en inglés -, que es el primer episodio de la segunda temporada de la icónica serie de ciencia ficción distópica Black Mirror.

Estimado lector, imagine usted que se le ofreciera la oportunidad de reemplazar a su ser querido fallecido por una persona que es mejor en cada aspecto, bajo tus propios estándares (buen sentido del humor, buen aspecto físico, fidelidad, etc.). ¿Aceptaría esta oportunidad? En este episodio de Black Mirror, Ash, la pareja de Martha, fallece en un accidente. Sola y angustiada, Martha descubre que está embarazada. Sumida en la desesperación, utiliza una tecnología emergente para reemplazar a Ash. Inicialmente, se comunica con este nuevo Ash (aquí le llamaremos AshBot) mediante mensajes de texto. Luego, puede conversar con AshBot mediante el celular. Finalmente, se encuentra cara a cara con un robot no metálico muy similar a Ash (AshBot).

Inicialmente, Martha se negaba de manera categórica a reemplazar a Ash mediante esta tecnología, pero esta idea cambió al descubrir que estaba embarazada. Así, Martha sucumbió ante la tentación de apaciguar el profundo dolor de perder a un ser querido fallecido mediante un sustituto. Sin embargo, a pesar de que AshBot era mejor que Ash en muchos aspectos, Martha termina decepcionándose. Esto hace que nos planteemos la pregunta de qué ha sucedido aquí. ¿Por qué es problemático reemplazar por versiones mejoradas a un ser querido fallecido si, después de todo, podría compartir las mismas cualidades con el ser querido original?

\section{Copias exactas}

Primer problema: si una persona quisiera un reemplazo de su ser querido, desearía que sea una copia exacta; sin embargo, AshBot no es una copia exacta de Ash. En el episodio, Martha recuerda constantemente que AshBot es muy diferente de Ash: AshBot no respira, no duerme, no come, no sangra, carece de voluntad, etc.; estas característi- cas desde luego, son perturbadoras e indeseables para Martha.

No obstante, hay ciertas cualidades mejoradas que Martha aprecia de AshBot. En palabras expresas de Martha, AshBot es muy bueno al momento de mantener relaciones sexuales, además, explícitamente señala que su aspecto físico luce como en los mejores días de Ash. Así, estas cualidades pueden ser vistas como un mejoramiento del Ash original. Sin embargo, estos mejoramientos son limitados, puesto que AshBot fue originado a partir de la información que Ash guardó en sus redes sociales, y esta información es limitada. En consecuencia, AshBot no comparte los mismos deseos, las mismas preferencias, la independencia, voluntad y asertividad de Ash. Esto último hace que Martha desprecie a AshBot $y$, después de todo, esto es entendible, dado que AshBot no es una copia exacta de Ash. Aunque suene redundante: AshBot no es Ash.

Ahora, para continuar la discusión filosófica, imaginemos que Martha pudiera tener un duplicado exacto de Ash. Este duplicado (llamémosle AshBotPro) tendría las mismas creencias, deseos, preferencias y recuerdos que Ash, incluso luciría y se comportaría exactamente como él. ¿Cuál sería el problema?

\section{Las propiedades relacionales importan}

Para responder esta última pregunta, tengamos en cuenta la diferencia entre propiedades intrínsecas y propiedades relacionales. Por un lado, una propiedad intrínseca es una propiedad que tiene un objeto en virtud solamente de sí mismo, es decir, esta propiedad no depende de su relación con otros objetos (el volumen de un cuerpo, por ejemplo, no depende de alguna relación con otro objeto). Por otro lado, una propiedad relacional depende, como el nombre lo sugiere, de la relación que un objeto tiene con otros (la propiedad de estar casado, por ejemplo, depende de la relación que tiene un marido con su esposa y viceversa).

Para que AshBotPro sea Ash, ambos deben tener las mismas propiedades relacionales; sin embargo, este no es el caso, puesto que "incluso los duplicados exactos no adquieren las cualidades relacionales del original" (Richards, 2020, p. 44). Para entender esta idea, veamos el siguiente ejemplo: un duplicado exacto de un billete no tiene las mismas propiedades relacionales que un billete original, puesto que la posesión de este duplicado no sería le- 
gal (para que lo sea, el billete duplicado debería ser producido, registrado y aprobado por la autoridad monetaria correspondiente). Así, esta es una propiedad relacional: un billete auténtico tiene la relación con la entidad monetaria correspondiente de haber sido producido, registrado y aprobado allí. En consecuencia, una copia exacta (una falsificación perfecta) no puede reproducir esta propiedad relacional. Precisamente esto es lo que sería AshBotPro: una falsificación perfecta. AshBotPro podría mantener todas las propiedades intrínsecas de Ash, mas no sus propiedades relacionales. Consideremos una propiedad relacional resaltante: el ser padre. El mero hecho de ser una copia exacta de Ash no hace que AshBotPro sea el padre de la hija de Martha - aunque es importante notar que sí podría mantener la relación, es decir, AshBotPro sí podría ser como Ash en la crianza de la hija de Martha, pero esta sería una nueva relación.

¿Realmente importa que AshBotPro y Ash no mantengan las mismas propiedades relacionales?, ¿no podrían Martha y los familiares de Ash estar satisfechos con un duplicado exacto de Ash? Después de todo, AshBotPro actuaría y luciría como Ash. Aquí, "la respuesta está en darse cuenta de que las propiedades relacionales importan" (Richards, 2020, p. 45). El amor, por ejemplo, es una propiedad relacional, al igual que el ser padre, y estas relaciones son importantes para las personas. Un duplicado exacto no puede conservar estas propiedades relacionales importantes. Así, esta tesis tiene como consecuencia que nuestros seres queridos son irremplazables, incluso por duplicados exactos, puesto que estos no mantendrían las mismas propiedades relacionales importantes.

Entonces, volviendo a la inicial discusión del transhumanismo, podemos obtener la siguiente conclusión: las tecnologías del mejoramiento humano no representan una amenaza a la identidad personal de un individuo en tanto que no alteran sus propiedades relacionales importantes; $y$, dado que es implausible suponer que los mejoramientos propuestos por el transhumanismo sucedan de una sola vez (el uso de estas tecnologías será gradual), esto hace prever que los individuos que usen estas tecnologías mantendrán sus propiedades relacionales importantes.

\section{Conclusiones}

A partir de todo lo expuesto, podemos obtener las siguientes conclusiones:

a) Los individuos que son personas no tienen características esenciales, es decir, no tienen capacidades y características fundamentales de la identidad de uno.

b) A pesar de la conclusión anterior, hay cualidades que realmente importan en el problema de la identidad personal: las propiedades relacionales. Esta tesis tiene como consecuencia que nuestros seres queridos son irremplazables, incluso por duplicados exactos.

c) El uso de las tecnologías de mejoramiento humano no representa una amenaza a la identidad personal de un individuo en tanto que no altera sus propiedades relacionales importantes.

\section{Referencias}

Annas, G., Andrews, L., \& Isasi, R. (2002). Protecting the endangered human: toward an international treaty prohibiting cloning and inheritable alterations. American Journal of Law and Medicine(28), 151-178.

https://doi.org/10.1017/S009885880001162X
Bostrom, N., \& Savulescu, J. (2009). Human enhancement. Oxford University Press.

DeGrazia, D. (2005). Enhancement Technologies and Human Identity. Journal of Medicine and Philosophy(30), 261-283. 
https://philosophy.columbian.gwu.edu/sites/g/files/zaxdzs1676/f/imag

e/degrazia enhancement.pdf

Elliott, C. (1999). A philosophical disease: Bioethics, culture and identity. Routledge.

Silva, J. (30 de Marzo de 2021). HUME Y EL

PROBLEMA DE LA IDENTIDAD PERSONAL 1: EL

IMPERSONALISMO/NIHILISMO [Video] . Youtube.

https://youtu.be/kn8lhU0yHAc

Mayr, E. (2004). What makes biology unique? Considerations on the
Autonomy of a Scientific Discipline. Cambridge University Press.

Okasha, S. (2019). Philosophy of Biology: A Very Short Introduction. Oxford University Press.

Richards, B. (2020). Be Right Back and Rejecting Tragedy: Would You Bring Back Your Deceased Loved One? En D. Johnson

(Ed.), Black Mirror and Philosophy: Dark Reflections (41-

49). Wiley-Blackwell.

Villena, D. (2010). ¿Yo soy una persona? Analítica(4), 55-

67. http://cesfia.org.pe/analitica/4/villena. $\underline{\text { pdf }}$

\section{Cómo citar este artículo:}

Silva, J. (2021). ¿Es el pensamiento transhumanista una amenaza a la dignidad humana? Futuro Hoy, 2(3), 29-35. https://doi.org/10.52749/fh.v2i3.5

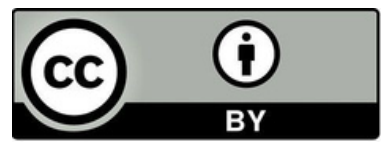

Esta obra está bajo licencia internacional Creative Commons 4.0 Reconocimiento 4.0. 